\title{
Positive solutions for a system of nonlinear Hadamard fractional differential equations involving coupled integral boundary conditions
}

\author{
Jiqiang Jiang ${ }^{1 *}\left(\mathbb{D}\right.$, Donal $\mathrm{O}^{\prime}$ Regan ${ }^{2}$, Jiafa $\mathrm{Xu}^{3}$ and Zhengqing $\mathrm{Fu}^{4}$
}

"Correspondence: qfiji@@163.com 'School of Mathematical Sciences, Qufu Normal University, Qufu, China Full list of author information is available at the end of the article

\begin{abstract}
In this paper we use the fixed point index to study the existence of positive solutions for a system of nonlinear Hadamard fractional differential equations involving coupled integral boundary conditions. Here we use appropriate nonnegative matrices to depict the coupling behavior for our nonlinearities.
\end{abstract}

MSC: 34B18; 34B10; 34B15

Keywords: Hadamard fractional differential equations; Integral boundary conditions; Positive solutions; Fixed point index

\section{Introduction}

In this paper we consider the system of nonlinear Hadamard fractional differential equations involving coupled integral boundary conditions

$$
\left\{\begin{array}{l}
D^{\beta} u(t)+f_{1}(t, u(t), v(t))=0, \quad 1<t<e, \\
D^{\beta} v(t)+f_{2}(t, u(t), v(t))=0, \quad 1<t<e, \\
u(1)=v(1)=u^{\prime}(1)=v^{\prime}(1)=0, \\
u(e)=\int_{1}^{e} h(s) v(s) \frac{d s}{s}, \\
v(e)=\int_{1}^{e} g(s) u(s) \frac{d s}{s},
\end{array}\right.
$$

where $\beta \in(2,3], D^{\beta}$ is the Hadamard fractional derivative of fractional order $\beta$, and $f_{i}$ $(i=1,2), h, g$ satisfy the following conditions:

(H1) $f_{i}(i=1,2)$ are nonnegative continuous functions on $[1, e] \times \mathbb{R}^{+} \times \mathbb{R}^{+}$,

(H2) $h, g \geq 0(\equiv \equiv)$ on $[1, e]$ with $\int_{1}^{e} h(t)(\log t)^{\beta-1} \frac{d t}{t} \cdot \int_{1}^{e} g(t)(\log t)^{\beta-1} \frac{d t}{t} \in(0,1)$.

Fractional-order differential equations is a rapidly developing area of research; we refer the reader to [1-48] and the references therein. In [1-9], the authors used iterative techniques to study existence and uniqueness of solutions for fractional boundary value problems. In [1] the authors studied positive solutions for the $p$-Laplacian fractional Riemann- 
Stieltjes integral boundary value problem

$$
\left\{\begin{array}{l}
-D_{t}^{\beta}\left(\varphi_{p}\left(-D_{t}^{\alpha} z\right)\right)(t)=f\left(t, z(t), D_{t}^{\gamma} z(t)\right), \quad t \in(0,1) \\
D_{t}^{\alpha} z(0)=D_{t}^{\alpha+1} z(0)=D_{t}^{\gamma} z(0)=0 \\
D_{t}^{\alpha} z(1)=0, \quad D_{t}^{\gamma} z(1)=\int_{0}^{1} D_{t}^{\gamma} z(s) d A(s),
\end{array}\right.
$$

where $D_{t}^{\alpha}, D_{t}^{\beta}, D_{t}^{\gamma}$ are the Riemann-Liouville fractional derivatives, and they not only obtained existence and uniqueness of positive solutions for (1.2), but also constructed an iteration sequence for the unique positive solution. In [10-32], the authors used fixed point methods to study the existence of (positive) solutions fractional order equations. In [10] Mawhin's continuation theorem was used to study the following fractional order boundary value problem at resonance:

$$
\left\{\begin{array}{l}
{ }^{c} D^{q} x(t)=f\left(t, x(t), x^{\prime}(t)\right), \quad t \in[0, T], \\
x(0)=\alpha I_{\eta}^{\gamma, \delta} x(\zeta), \quad x(T)=\beta^{\rho} I^{p} x(\xi), \quad 0<\zeta, \xi \leq T,
\end{array}\right.
$$

where ${ }^{c} D^{q}$ is the Caputo fractional derivative, $I_{\eta}^{\gamma, \delta}$ is a Erdélyi-Kober type integral, and ${ }^{\rho} I^{p}$ denotes the generalized Riemann-Liouville type integral boundary conditions. For fractional differential systems, see [23-32]. In [23], using the Leray-Schauder alternative and the Banach contraction principle, the authors studied existence and uniqueness of solutions for the system of nonlinear Caputo type sequential fractional integro-differential equations

$$
\left\{\begin{array}{l}
\left({ }^{c} D^{\alpha}+\lambda^{c} D^{\alpha-1}\right) u(t)=f\left(t, u(t), v(t),{ }^{c} D^{p_{1}} v(t), I^{q_{1}} v(t)\right), \quad t \in(0,1), \\
\left({ }^{c} D^{\beta}+\mu^{c} D^{\beta-1}\right) v(t)=g\left(t, u(t),{ }^{c} D^{p_{2}} u(t), I^{q_{2}} u(t), v(t)\right), \quad t \in(0,1), \\
u(0)=u^{\prime}(0)=u^{\prime \prime}(0)=0, \quad u(1)=\int_{0}^{1} u(s) d H_{1}(s)+\int_{0}^{1} v(s) d H_{2}(s), \\
v(0)=v^{\prime}(0)=v^{\prime \prime}(0)=0, \quad v(1)=\int_{0}^{1} u(s) d K_{1}(s)+\int_{0}^{1} v(s) d K_{2}(s)
\end{array}\right.
$$

Hadamard fractional differential equations are also popular in the literature; see [33-48] and the references therein. In [33], the authors used the Banach contraction principle, the Leray-Schauder's alternative, and Krasnoselskii's fixed-point theorem to study the existence and uniqueness of solutions for the coupled system of nonlinear sequential Caputo and Hadamard fractional differential equations with coupled separated boundary conditions

$$
\begin{cases}{ }^{C} D^{p_{1} H} D^{q_{1}} x(t)=f(t, x(t), y(t)), & t \in[a, b], \\ { }^{H} D^{q_{2} C} D^{p_{2}} x(t)=g(t, x(t), y(t)), & t \in[a, b], \\ \alpha_{1} x(a)+\alpha_{2}{ }^{C} D^{p_{2}} y(a)=0, & \beta_{1} x(b)+\beta_{2}{ }^{C} D^{p_{2}} y(b)=0, \\ \alpha_{3} y(a)+\alpha_{4}{ }^{H} D^{q_{1}} x(a)=0, & \beta_{3} y(b)+\beta_{4}{ }^{H} D^{q_{1}} x(b)=0,\end{cases}
$$

where ${ }^{C} D^{p_{i}},{ }^{H} D^{q_{i}}$ are respectively the Caputo and Hadamard fractional derivatives. In [34] the authors established positive solutions for the coupled Hadamard fractional integral 
boundary value problems

$$
\left\{\begin{array}{l}
D^{\alpha} u(t)+\lambda f(t, u(t), v(t))=0, \quad t \in(1, e), \lambda>0 \\
D^{\beta} v(t)+\lambda g(t, u(t), v(t))=0, \quad t \in(1, e), \lambda>0 \\
u^{(j)}(1)=v^{(j)}(1)=0, \quad 0 \leq j \leq n-2, \\
u(e)=\mu \int_{1}^{e} v(s) \frac{d s}{s} \\
v(e)=v \int_{1}^{e} u(s) \frac{d s}{s}
\end{array}\right.
$$

where $\alpha, \beta \in(n-1, n]$ and $n \geq 3, D^{\alpha}, D^{\beta}$ are the Hadamard fractional derivatives and their nonlinearities $f, g$ satisfy the following conditions:

$(\mathrm{H})_{\text {Yang1 }}$ There exists $\left[\theta_{1}, \theta_{2}\right] \subset(1, e)$ such that $\liminf _{u \rightarrow+\infty} \min _{t \in\left[\theta_{1}, \theta_{2}\right]} \frac{f(t, u, v)}{u}=+\infty$ and $\liminf _{v \rightarrow+\infty} \min _{t \in\left[\theta_{1}, \theta_{2}\right]} \frac{g(t, u, v)}{v}=+\infty$;

or

$(\mathrm{H})_{\text {Yang2 }}$ There exists $\left[\theta_{1}, \theta_{2}\right] \subset(1, e)$ such that $\liminf _{v \rightarrow+\infty} \min _{t \in\left[\theta_{1}, \theta_{2}\right]} \frac{f(t, u, v)}{v}=+\infty$ and $\liminf _{u \rightarrow+\infty} \min _{t \in\left[\theta_{1}, \theta_{2}\right]} \frac{g(t, u, v)}{u}=+\infty$.

Motivated by the above, in this paper we study the existence of positive solutions for the system of nonlinear Hadamard fractional differential equations (1.1) involving coupled integral boundary conditions. We use appropriate nonnegative matrices to depict the coupling behavior for our nonlinearities, and note that they can grow both superlinearly and sublinearly. We remark here that our conditions for nonlinear terms are not as restrictive as those in $(\mathrm{H})_{\text {Yang1 }}$ and $(\mathrm{H})_{\text {Yang2 }}$; see $(\mathrm{H} 3)-(\mathrm{H} 6)$ in Sect. 3.

\section{Preliminaries}

In this section, we first provide some material for Hadamard fractional calculus; for details, see the book [49].

Definition 2.1 The Hadamard derivative of fractional order $q$ for a function $g:[1, \infty) \rightarrow$ $\mathbb{R}$ is defined as

$$
D^{q} g(t)=\frac{1}{\Gamma(n-q)}\left(t \frac{d}{d t}\right)^{n} \int_{1}^{t}(\log t-\log s)^{n-q-1} g(s) \frac{d s}{s}, \quad n-1<q<n,
$$

where $n=[q]+1 ;[q]$ denotes the integer part of the real number $q$ and $\log (\cdot)=\log _{e}(\cdot)$.

Definition 2.2 The Hadamard fractional integral of order $q$ for a function $g:[1, \infty) \rightarrow \mathbb{R}$ is defined as

$$
I^{q} g(t)=\frac{1}{\Gamma(q)} \int_{1}^{t}(\log t-\log s)^{q-1} g(s) \frac{d s}{s}, \quad q>0,
$$

provided the integral exists.

In what follows, we calculate the Green's functions associated with (1.1) and study some properties of these Green's functions. 
Lemma 2.3 (see [34, Lemma 2.3]) Let $x, y \in C[1, e]$. Then the integral boundary value problem

$$
\left\{\begin{array}{l}
D^{\beta} u(t)+x(t)=0, \quad D^{\beta} v(t)+y(t)=0, \quad t \in(1, e), \\
u(1)=v(1)=u^{\prime}(1)=v^{\prime}(1)=0, \\
u(e)=\int_{1}^{e} h(s) v(s) \frac{d s}{s} \\
v(e)=\int_{1}^{e} g(s) u(s) \frac{d s}{s}
\end{array}\right.
$$

can be transformed into the following Hammerstein type integral equations:

$$
\left\{\begin{aligned}
u(t)= & \int_{1}^{e} G_{1}(t, s) x(s) \frac{d s}{s}+\frac{d_{h}(\log t)^{\beta-1}}{d_{g, h} \Gamma(\beta)} \int_{1}^{e} \int_{1}^{e} g(t) G_{1}(t, s) \frac{d t}{t} x(s) \frac{d s}{s} \\
& +\frac{(\log t)^{\beta-1}}{d_{g, h} \Gamma(\beta)} \int_{1}^{e} \int_{1}^{e} h(t) G_{1}(t, s) \frac{d t}{t} y(s) \frac{d s}{s}, \\
v(t)= & \int_{1}^{e} G_{1}(t, s) y(s) \frac{d s}{s}+\frac{d_{g}(\log t)^{\beta-1}}{d_{g, h} \Gamma(\beta)} \int_{1}^{e} \int_{1}^{e} h(t) G_{1}(t, s) \frac{d t}{t} y(s) \frac{d s}{s} \\
& +\frac{(\log t)^{\beta-1}}{d_{g, h} \Gamma(\beta)} \int_{1}^{e} \int_{1}^{e} g(t) G_{1}(t, s) \frac{d t}{t} x(s) \frac{d s}{s},
\end{aligned}\right.
$$

where

$$
G_{1}(t, s)=\frac{1}{\Gamma(\beta)} \begin{cases}(\log t)^{\beta-1}(1-\log s)^{\beta-1}-(\log t-\log s)^{\beta-1}, & 1 \leq s \leq t \leq e, \\ (\log t)^{\beta-1}(1-\log s)^{\beta-1}, & 1 \leq t \leq s \leq e\end{cases}
$$

here, $d_{g, h}, d_{g}, d_{h}$ are three positive constants defined in the proof.

Proof From Lemma 2.3 of [34] we have

$$
\begin{aligned}
& u(t)=c_{11}(\log t)^{\beta-1}+c_{12}(\log t)^{\beta-2}+c_{13}(\log t)^{\beta-3}-\frac{1}{\Gamma(\beta)} \int_{1}^{t}(\log t-\log s)^{\beta-1} x(s) \frac{d s}{s}, \\
& v(t)=c_{21}(\log t)^{\beta-1}+c_{22}(\log t)^{\beta-2}+c_{23}(\log t)^{\beta-3}-\frac{1}{\Gamma(\beta)} \int_{1}^{t}(\log t-\log s)^{\beta-1} y(s) \frac{d s}{s},
\end{aligned}
$$

where $c_{1 i}, c_{2 i} \in \mathbb{R}, i=1,2,3$. Note that $u(1)=v(1)=u^{\prime}(1)=v^{\prime}(1)=0$ implies $c_{12}, c_{13}, c_{22}, c_{23}=$ 0 . Then we have

$$
\begin{aligned}
& u(t)=c_{11}(\log t)^{\beta-1}-\frac{1}{\Gamma(\beta)} \int_{1}^{t}(\log t-\log s)^{\beta-1} x(s) \frac{d s}{s}, \\
& v(t)=c_{21}(\log t)^{\beta-1}-\frac{1}{\Gamma(\beta)} \int_{1}^{t}(\log t-\log s)^{\beta-1} y(s) \frac{d s}{s} .
\end{aligned}
$$

Using the conditions $u(e)=\int_{1}^{e} h(s) v(s) \frac{d s}{s}, v(e)=\int_{1}^{e} g(s) u(s) \frac{d s}{s}$, we obtain

$$
\begin{aligned}
c_{11} & -\frac{1}{\Gamma(\beta)} \int_{1}^{e}(1-\log s)^{\beta-1} x(s) \frac{d s}{s} \\
= & c_{21} \int_{1}^{e} h(t)(\log t)^{\beta-1} \frac{d t}{t}-\frac{1}{\Gamma(\beta)} \int_{1}^{e} h(t) \int_{1}^{t}(\log t-\log s)^{\beta-1} y(s) \frac{d s}{s} \frac{d t}{t}, \\
c_{21} & -\frac{1}{\Gamma(\beta)} \int_{1}^{e}(1-\log s)^{\beta-1} y(s) \frac{d s}{s} \\
= & c_{11} \int_{1}^{e} g(t)(\log t)^{\beta-1} \frac{d t}{t}-\frac{1}{\Gamma(\beta)} \int_{1}^{e} g(t) \int_{1}^{t}(\log t-\log s)^{\beta-1} x(s) \frac{d s}{s} \frac{d t}{t} .
\end{aligned}
$$


This implies that

$$
\begin{gathered}
{\left[\begin{array}{cc}
1 & -\int_{1}^{e} h(t)(\log t)^{\beta-1} \frac{d t}{t} \\
-\int_{1}^{e} g(t)(\log t)^{\beta-1} \frac{d t}{t} & 1
\end{array}\right]\left[\begin{array}{l}
c_{11} \\
c_{21}
\end{array}\right]} \\
=\left[\begin{array}{l}
\frac{1}{\Gamma(\beta)} \int_{1}^{e}(1-\log s)^{\beta-1} x(s) \frac{d s}{s}-\frac{1}{\Gamma(\beta)} \int_{1}^{e} h(t) \int_{1}^{t}(\log t-\log s)^{\beta-1} y(s) \frac{d s}{s} \frac{d t}{t} \\
\frac{1}{\Gamma(\beta)} \int_{1}^{e}(1-\log s)^{\beta-1} y(s) \frac{d s}{s}-\frac{1}{\Gamma(\beta)} \int_{1}^{e} g(t) \int_{1}^{t}(\log t-\log s)^{\beta-1} x(s) \frac{d s}{s} \frac{d t}{t}
\end{array}\right] .
\end{gathered}
$$

Let $d_{g, h}=1-\int_{1}^{e} h(t)(\log t)^{\beta-1} \frac{d t}{t} \cdot \int_{1}^{e} g(t)(\log t)^{\beta-1} \frac{d t}{t}, \quad d_{h}=\int_{1}^{e} h(t)(\log t)^{\beta-1} \frac{d t}{t}, \quad d_{g}=$ $\int_{1}^{e} g(t)(\log t)^{\beta-1} \frac{d t}{t}$. Then

$$
\begin{aligned}
{\left[\begin{array}{l}
c_{11} \\
c_{21}
\end{array}\right]=} & \frac{1}{d_{g, h}}\left[\begin{array}{cc}
1 & \int_{1}^{e} h(t)(\log t)^{\beta-1} \frac{d t}{t} \\
\int_{1}^{e} g(t)(\log t)^{\beta-1} \frac{d t}{t} & 1
\end{array}\right] \\
& \cdot\left[\begin{array}{l}
\frac{1}{\Gamma(\beta)} \int_{1}^{e}(1-\log s)^{\beta-1} x(s) \frac{d s}{s}-\frac{1}{\Gamma(\beta)} \int_{1}^{e} h(t) \int_{1}^{t}(\log t-\log s)^{\beta-1} y(s) \frac{d s}{s} \frac{d t}{t} \\
\frac{1}{\Gamma(\beta)} \int_{1}^{e}(1-\log s)^{\beta-1} y(s) \frac{d s}{s}-\frac{1}{\Gamma(\beta)} \int_{1}^{e} g(t) \int_{1}^{t}(\log t-\log s)^{\beta-1} x(s) \frac{d s}{s} \frac{d t}{t}
\end{array}\right] .
\end{aligned}
$$

Consequently, we have

$$
\begin{aligned}
& u(t)=\frac{(\log t)^{\beta-1}}{d_{g, h} \Gamma(\beta)} \int_{1}^{e}(1-\log s)^{\beta-1} x(s) \frac{d s}{s} \\
& -\frac{(\log t)^{\beta-1}}{d_{g, h} \Gamma(\beta)} \int_{1}^{e} h(t) \int_{1}^{t}(\log t-\log s)^{\beta-1} y(s) \frac{d s}{s} \frac{d t}{t} \\
& +\frac{d_{h}(\log t)^{\beta-1}}{d_{g, h} \Gamma(\beta)} \int_{1}^{e}(1-\log s)^{\beta-1} y(s) \frac{d s}{s} \\
& -\frac{d_{h}(\log t)^{\beta-1}}{d_{g, h} \Gamma(\beta)} \int_{1}^{e} g(t) \int_{1}^{t}(\log t-\log s)^{\beta-1} x(s) \frac{d s}{s} \frac{d t}{t} \\
& -\frac{1}{\Gamma(\beta)} \int_{1}^{t}(\log t-\log s)^{\beta-1} x(s) \frac{d s}{s} \\
& =\frac{(\log t)^{\beta-1}}{d_{g, h} \Gamma(\beta)} \int_{1}^{e} h(t)(\log t)^{\beta-1} \frac{d t}{t} \int_{1}^{e}(1-\log s)^{\beta-1} y(s) \frac{d s}{s} \\
& -\frac{(\log t)^{\beta-1}}{d_{g, h} \Gamma(\beta)} \int_{1}^{e} h(t) \int_{1}^{t}(\log t-\log s)^{\beta-1} y(s) \frac{d s}{s} \frac{d t}{t} \\
& +\frac{(\log t)^{\beta-1}}{d_{g, h} \Gamma(\beta)} \int_{1}^{e}(1-\log s)^{\beta-1} x(s) \frac{d s}{s} \\
& -\frac{d_{h}(\log t)^{\beta-1}}{d_{g, h} \Gamma(\beta)} \int_{1}^{e} g(t) \int_{1}^{t}(\log t-\log s)^{\beta-1} x(s) \frac{d s}{s} \frac{d t}{t} \\
& -\frac{1}{\Gamma(\beta)} \int_{1}^{t}(\log t-\log s)^{\beta-1} x(s) \frac{d s}{s}+\frac{(\log t)^{\beta-1}}{\Gamma(\beta)} \int_{1}^{e}(1-\log s)^{\beta-1} x(s) \frac{d s}{s} \\
& -\frac{(\log t)^{\beta-1}}{\Gamma(\beta)} \int_{1}^{e}(1-\log s)^{\beta-1} x(s) \frac{d s}{s} \\
& =\int_{1}^{e} G_{1}(t, s) x(s) \frac{d s}{s}+\frac{d_{h}(\log t)^{\beta-1}}{d_{g, h} \Gamma(\beta)}\left[\int_{1}^{e} g(t)(\log t)^{\beta-1} \frac{d t}{t} \int_{1}^{e}(1-\log s)^{\beta-1} x(s) \frac{d s}{s}\right. \\
& \left.-\int_{1}^{e} g(t) \int_{1}^{t}(\log t-\log s)^{\beta-1} x(s) \frac{d s}{s} \frac{d t}{t}\right]
\end{aligned}
$$




$$
\begin{aligned}
& +\frac{(\log t)^{\beta-1}}{d_{g, h} \Gamma(\beta)}\left[\int_{1}^{e} h(t)(\log t)^{\beta-1} \frac{d t}{t} \int_{1}^{e}(1-\log s)^{\beta-1} y(s) \frac{d s}{s}\right. \\
& \left.-\int_{1}^{e} h(t) \int_{1}^{t}(\log t-\log s)^{\beta-1} y(s) \frac{d s}{s} \frac{d t}{t}\right] \\
& =\int_{1}^{e} G_{1}(t, s) x(s) \frac{d s}{s}+\frac{d_{h}(\log t)^{\beta-1}}{d_{g, h} \Gamma(\beta)}\left[\int_{1}^{e} g(t)(\log t)^{\beta-1} \frac{d t}{t} \int_{1}^{e}(1-\log s)^{\beta-1} x(s) \frac{d s}{s}\right. \\
& \left.-\int_{1}^{e} g(t) \int_{s}^{e}(\log t-\log s)^{\beta-1} x(s) \frac{d t}{t} \frac{d s}{s}\right] \\
& +\frac{(\log t)^{\beta-1}}{d_{g, h} \Gamma(\beta)}\left[\int_{1}^{e} h(t)(\log t)^{\beta-1} \frac{d t}{t} \int_{1}^{e}(1-\log s)^{\beta-1} y(s) \frac{d s}{s}\right. \\
& \left.-\int_{1}^{e} h(t) \int_{s}^{e}(\log t-\log s)^{\beta-1} y(s) \frac{d t}{t} \frac{d s}{s}\right] \\
& =\int_{1}^{e} G_{1}(t, s) x(s) \frac{d s}{s}+\frac{d_{h}(\log t)^{\beta-1}}{d_{g, h} \Gamma(\beta)} \int_{1}^{e} \int_{1}^{e} g(t) G_{1}(t, s) \frac{d t}{t} x(s) \frac{d s}{s} \\
& +\frac{(\log t)^{\beta-1}}{d_{g, h} \Gamma(\beta)} \int_{1}^{e} \int_{1}^{e} h(t) G_{1}(t, s) \frac{d t}{t} y(s) \frac{d s}{s} .
\end{aligned}
$$

Similarly, we also obtain that

$$
\begin{aligned}
v(t)= & c_{21}(\log t)^{\beta-1}-\frac{1}{\Gamma(\beta)} \int_{1}^{t}(\log t-\log s)^{\beta-1} y(s) \frac{d s}{s} \\
& +\frac{1}{\Gamma(\beta)} \int_{1}^{e}(\log t)^{\beta-1}(1-\log s)^{\beta-1} y(s) \frac{d s}{s} \\
& -\frac{1}{\Gamma(\beta)} \int_{1}^{e}(\log t)^{\beta-1}(1-\log s)^{\beta-1} y(s) \frac{d s}{s} \\
= & \int_{1}^{e} G_{1}(t, s) y(s) \frac{d s}{s}-\frac{1}{\Gamma(\beta)} \int_{1}^{e}(\log t)^{\beta-1}(1-\log s)^{\beta-1} y(s) \frac{d s}{s} \\
& +\frac{d_{g}(\log t)^{\beta-1}}{d_{g, h} \Gamma(\beta)} \int_{1}^{e}(1-\log s)^{\beta-1} x(s) \frac{d s}{s} \\
& -\frac{d_{g}(\log t)^{\beta-1}}{d_{g, h} \Gamma(\beta)} \int_{1}^{e} h(t) \int_{1}^{t}(\log t-\log s)^{\beta-1} y(s) \frac{d s}{s} \frac{d t}{t} \\
& +\frac{(\log t)^{\beta-1}}{d_{g, h} \Gamma(\beta)} \int_{1}^{e}(1-\log s)^{\beta-1} y(s) \frac{d s}{s} \\
& -\frac{(\log t)^{\beta-1}}{d_{g, h} \Gamma(\beta)} \int_{1}^{e} g(t) \int_{1}^{t}(\log t-\log s)^{\beta-1} x(s) \frac{d s}{s} \frac{d t}{t} \\
= & \int_{1}^{e} G_{1}(t, s) y(s) \frac{d s}{s}+\frac{d_{g}(\log t)^{\beta-1}}{d_{g, h} \Gamma(\beta)}\left[\int_{1}^{e} h(t)(\log t)^{\beta-1} \frac{d t}{t} \int_{1}^{e}(1-\log s)^{\beta-1} y(s) \frac{d s}{s}\right. \\
& \left.-\int_{1}^{e} h(t) \int_{1}^{t}(\log t-\log s)^{\beta-1} y(s) \frac{d s}{s} \frac{d t}{t}\right] \\
& +\frac{(\log t)^{\beta-1}}{d_{g, h} \Gamma(\beta)}\left[\int_{1}^{e} g(t)(\log t)^{\beta-1} \frac{d t}{t} \int_{1}^{e}(1-\log s)^{\beta-1} x(s) \frac{d s}{s}\right. \\
& \left.-\int_{1}^{e} g(t) \int_{1}^{t}(\log t-\log s)^{\beta-1} x(s) \frac{d s}{s} \frac{d t}{t}\right] \\
&
\end{aligned}
$$




$$
\begin{aligned}
= & \int_{1}^{e} G_{1}(t, s) y(s) \frac{d s}{s}+\frac{d_{g}(\log t)^{\beta-1}}{d_{g, h} \Gamma(\beta)} \int_{1}^{e} \int_{1}^{e} h(t) G_{1}(t, s) \frac{d t}{t} y(s) \frac{d s}{s} \\
& +\frac{(\log t)^{\beta-1}}{d_{g, h} \Gamma(\beta)} \int_{1}^{e} \int_{1}^{e} g(t) G_{1}(t, s) \frac{d t}{t} x(s) \frac{d s}{s} .
\end{aligned}
$$

This completes the proof.

From Lemma 2.3, we note (1.1) is equivalent to the Hammerstein type integral equations

$$
\left\{\begin{aligned}
u(t)= & \int_{1}^{e} G_{1}(t, s) f_{1}(s, u(s), v(s)) \frac{d s}{s} \\
& +\frac{d_{h}(\log t)^{\beta-1}}{d_{g, h} \Gamma(\beta)} \int_{1}^{e} \int_{1}^{e} g(t) G_{1}(t, s) \frac{d t}{t} f_{1}(s, u(s), v(s)) \frac{d s}{s} \\
& +\frac{\left(\log t t^{\beta-1}\right.}{d_{g, h} \Gamma(\beta)} \int_{1}^{e} \int_{1}^{e} h(t) G_{1}(t, s) \frac{d t}{t} f_{2}(s, u(s), v(s)) \frac{d s}{s}, \\
v(t)= & \int_{1}^{e} G_{1}(t, s) f_{2}(s, u(s), v(s)) \frac{d s}{s} \\
& +\frac{d_{g}(\log t)^{\beta-1}}{d_{g, h} \Gamma(\beta)} \int_{1}^{e} \int_{1}^{e} h(t) G_{1}(t, s) \frac{d t}{t} f_{2}(s, u(s), v(s)) \frac{d s}{s} \\
& +\frac{(\log t)^{\beta-1}}{d_{g, h} \Gamma(\beta)} \int_{1}^{e} \int_{1}^{e} g(t) G_{1}(t, s) \frac{d t}{t} f_{1}(s, u(s), v(s)) \frac{d s}{s} .
\end{aligned}\right.
$$

Lemma 2.4 The function $G_{1}(t, s)$ satisfies the following inequalities:

(I1) $\frac{1}{\Gamma(\beta)}(\log t)^{\beta-1}(1-\log t) \log s(1-\log s)^{\beta-1} \leq G_{1}(t, s) \leq \frac{\beta-1}{\Gamma(\beta)} \log s(1-\log s)^{\beta-1}$ for $t, s \in[1, e]$,

(I2) $\frac{1}{\Gamma(\beta)}(\log t)^{\beta-1}(1-\log t) \log s(1-\log s)^{\beta-1} \leq G_{1}(t, s) \leq \frac{\beta-1}{\Gamma(\beta)}(\log t)^{\beta-1}(1-\log t)$ for $t, s \in[1, e]$.

Proof We note a result from [14]. Let $\beta \in(n-1, n]$ with $n \in \mathbb{N}, n \geq 3$. Then the function

$$
G(z, l)=\frac{1}{\Gamma(\beta)} \begin{cases}z^{\beta-1}(1-l)^{\beta-1}-(z-l)^{\beta-1}, & 0 \leq l \leq z \leq 1 \\ z^{\beta-1}(1-l)^{\beta-1}, & 0 \leq z \leq l \leq 1\end{cases}
$$

has the following properties:

(R1) $G(z, l)=G(1-l, 1-z)$ for $z, l \in[0,1]$;

(R2) $\Gamma(\beta) k(z) q(l) \leq G(z, l) \leq(\beta-1) q(l)$ for $z, l \in[0,1]$;

(R3) $\Gamma(\beta) k(z) q(l) \leq G(z, l) \leq(\beta-1) k(z)$ for $z, l \in[0,1]$, where $k(z)=\frac{z^{\beta-1}(1-z)}{\Gamma(\beta)}$, $q(l)=\frac{l(1-l)^{\beta-1}}{\Gamma(\beta)}$.

Now, we turn our attention to $G_{1}$. If $\log t, \log s$ are regarded as $z$, $l$, then from (R2), (R3) we have

$$
\begin{aligned}
& \Gamma(\beta) k(\log t) q(\log s) \leq G(\log t, \log s) \leq(\beta-1) q(\log s), \\
& \Gamma(\beta) k(\log t) q(\log s) \leq G(\log t, \log s) \leq(\beta-1) k(\log t), \quad \text { for } t, s \in[1, e] .
\end{aligned}
$$

Thus (I1), (I2) hold. This completes the proof.

Let $\mu(t)=\frac{1}{\Gamma(\beta)} \log t(1-\log t)^{\beta-1}$ for $t \in[1, e]$.

Lemma 2.5 Let $\kappa_{1}=\frac{\beta^{2} \Gamma(\beta)}{\Gamma(2 \beta+2)}, \kappa_{2}=\frac{\beta-1}{\Gamma(\beta+2)}$. Then, for any $s \in[1, e]$, the following inequalities hold:

$$
\kappa_{1} \mu(s) \leq \int_{1}^{e} G_{1}(t, s) \mu(t) \frac{d t}{t} \leq \kappa_{2} \mu(s) .
$$


This is a direct result from Lemma 2.4(I1), so we omit its proof.

Let $E:=C[1, e],\|u\|:=\max _{t \in[1, e]}|u(t)|, P:=\{u \in E: u(t) \geq 0, \forall t \in[1, e]\}$. Then $(E,\|\cdot\|)$ is a real Banach space and $P$ is a cone on $E$. From Lemma 2.3 and (2.4), we define operators $A_{i}: P \times P \rightarrow P$ as follows:

$$
\left\{\begin{aligned}
A_{1}(u, v)(t)= & \int_{1}^{e} G_{1}(t, s) f_{1}(s, u(s), v(s)) \frac{d s}{s} \\
& +\frac{d_{h}(\log t)^{\beta-1}}{d_{g, h} \Gamma(\beta)} \int_{1}^{e} \int_{1}^{e} g(t) G_{1}(t, s) \frac{d t}{t} f_{1}(s, u(s), v(s)) \frac{d s}{s} \\
& +\frac{(\log t)^{\beta-1}}{d_{g, h} \Gamma(\beta)} \int_{1}^{e} \int_{1}^{e} h(t) G_{1}(t, s) \frac{d t}{t} f_{2}(s, u(s), v(s)) \frac{d s}{s}, \\
A_{2}(u, v)(t)= & \int_{1}^{e} G_{1}(t, s) f_{2}(s, u(s), v(s)) \frac{d s}{s} \\
& +\frac{d_{g}(\log t)^{\beta-1}}{d_{g, h} \Gamma(\beta)} \int_{1}^{e} \int_{1}^{e} h(t) G_{1}(t, s) \frac{d t}{t} f_{2}(s, u(s), v(s)) \frac{d s}{s} \\
& +\frac{(\log t)^{\beta-1}}{d_{g, h} \Gamma(\beta)} \int_{1}^{e} \int_{1}^{e} g(t) G_{1}(t, s) \frac{d t}{t} f_{1}(s, u(s), v(s)) \frac{d s}{s},
\end{aligned}\right.
$$

and

$$
A(u, v)(t)=\left(A_{1}(u, v), A_{2}(u, v)\right)(t) \quad \text { for } t \in[1, e] .
$$

Note $A_{i}: P \times P \rightarrow P, A: P \times P \rightarrow P \times P$ are completely continuous operators and $(u, v)$ solves (1.1) if and only if $(u, v)$ is a fixed point of the operator $A$.

Lemma 2.6 Let $P_{0}=\left\{z \in P: z(t) \geq \frac{(\log t)^{\beta-1}(1-\log t)}{\beta-1}\|z\|, \forall t \in[1, e]\right\}$. Then $P_{0}$ is also a cone on $E$, and $A_{i}(P \times P) \subset P_{0}, i=1,2$.

Proof We only prove $A_{1}(P \times P) \subset P_{0}$. From Lemma 2.4(I1), for $t \in[1, e]$, we have

$$
\begin{aligned}
A_{1}(u, v)(t)= & \int_{1}^{e} G_{1}(t, s) f_{1}(s, u(s), v(s)) \frac{d s}{s} \\
& +\frac{d_{h}(\log t)^{\beta-1}}{d_{g, h} \Gamma(\beta)} \int_{1}^{e} \int_{1}^{e} g(t) G_{1}(t, s) \frac{d t}{t} f_{1}(s, u(s), v(s)) \frac{d s}{s} \\
& +\frac{(\log t)^{\beta-1}}{d_{g, h} \Gamma(\beta)} \int_{1}^{e} \int_{1}^{e} h(t) G_{1}(t, s) \frac{d t}{t} f_{2}(s, u(s), v(s)) \frac{d s}{s} \\
\leq & \int_{1}^{e} \frac{\beta-1}{\Gamma(\beta)} \log s(1-\log s)^{\beta-1} f_{1}(s, u(s), v(s)) \frac{d s}{s} \\
& +\frac{d_{h}}{d_{g, h} \Gamma(\beta)} \int_{1}^{e} \int_{1}^{e} g(t) G_{1}(t, s) \frac{d t}{t} f_{1}(s, u(s), v(s)) \frac{d s}{s} \\
& +\frac{1}{d_{g, h} \Gamma(\beta)} \int_{1}^{e} \int_{1}^{e} h(t) G_{1}(t, s) \frac{d t}{t} f_{2}(s, u(s), v(s)) \frac{d s}{s}
\end{aligned}
$$

and

$$
\begin{aligned}
A_{1}(u, v)(t) \geq & \int_{1}^{e} \frac{(\log t)^{\beta-1}(1-\log t)}{\Gamma(\beta)} \log s(1-\log s)^{\beta-1} f_{1}(s, u(s), v(s)) \frac{d s}{s} \\
& +\frac{d_{h}(\log t)^{\beta-1}(1-\log t)}{d_{g, h} \Gamma(\beta)} \int_{1}^{e} \int_{1}^{e} g(t) G_{1}(t, s) \frac{d t}{t} f_{1}(s, u(s), v(s)) \frac{d s}{s} \\
& +\frac{(\log t)^{\beta-1}(1-\log t)}{d_{g, h} \Gamma(\beta)} \int_{1}^{e} \int_{1}^{e} h(t) G_{1}(t, s) \frac{d t}{t} f_{2}(s, u(s), v(s)) \frac{d s}{s}
\end{aligned}
$$


Note that $\beta-1>1$, so we have

$$
A_{1}(u, v)(t) \geq \frac{(\log t)^{\beta-1}(1-\log t)}{\beta-1}\left\|A_{1}(u, v)\right\| \quad \text { for } u, v \in P, t \in[1, e]
$$

This completes the proof.

Lemma 2.7 (see [50]) Let E be a real Banach space and $P$ be a cone on E. Suppose that $\Omega \subset E$ is a bounded open set and that $A: \bar{\Omega} \cap P \rightarrow P$ is a continuous compact operator. If there exists $\omega_{0} \in P \backslash\{0\}$ such that

$$
\omega-A \omega \neq \lambda \omega_{0}, \quad \forall \lambda \geq 0, \omega \in \partial \Omega \cap P,
$$

then $i(A, \Omega \cap P, P)=0$, where $i$ denotes the fixed point index on $P$.

Lemma 2.8 (see [50]) Let E be a real Banach space and $P$ be a cone on E. Suppose that $\Omega \subset E$ is a bounded open set with $0 \in \Omega$ and that $A: \bar{\Omega} \cap P \rightarrow P$ is a continuous compact operator. If

$$
\omega-\lambda A \omega \neq 0, \forall \lambda \in[0,1], \omega \in \partial \Omega \cap P,
$$

then $i(A, \Omega \cap P, P)=1$.

\section{Main results}

Let

$$
\begin{aligned}
& \kappa_{3}=\frac{\beta}{d_{g, h} \Gamma(2 \beta+1)} \int_{1}^{e} g(t)(\log t)^{\beta-1}(1-\log t) \frac{d t}{t}, \\
& \kappa_{4}=\frac{\beta}{d_{g, h} \Gamma(2 \beta+1)} \int_{1}^{e} h(t)(\log t)^{\beta-1}(1-\log t) \frac{d t}{t}, \\
& \kappa_{5}=\frac{\beta(\beta-1)}{d_{g, h} \Gamma(2 \beta+1)} \int_{1}^{e} g(t) \frac{d t}{t}, \quad \kappa_{6}=\frac{\beta(\beta-1)}{d_{g, h} \Gamma(2 \beta+1)} \int_{1}^{e} h(t) \frac{d t}{t} .
\end{aligned}
$$

Now we list our assumptions for the nonlinearities $f_{i}(i=1,2)$.

(H3) There are $a_{1 i}, b_{1 i} \geq 0(i=1,2)$ and $l_{1}, l_{2}>0$ such that

$$
\begin{aligned}
& a_{11}\left(\kappa_{1}+\kappa_{3} d_{h}\right)+a_{12} \kappa_{4}<1, \quad b_{12}\left(\kappa_{1}+d_{g} \kappa_{4}\right)+b_{11} \kappa_{3}<1, \\
& \operatorname{det}\left(\begin{array}{cc}
b_{11}\left(\kappa_{1}+\kappa_{3} d_{h}\right)+b_{12} \kappa_{4} & a_{11}\left(\kappa_{1}+\kappa_{3} d_{h}\right)+a_{12} \kappa_{4}-1 \\
b_{12}\left(\kappa_{1}+d_{g} \kappa_{4}\right)+b_{11} \kappa_{3}-1 & a_{12}\left(\kappa_{1}+d_{g} \kappa_{4}\right)+a_{11} \kappa_{3}
\end{array}\right)>0, \\
& \left(\begin{array}{l}
f_{1}(t, x, y) \\
f_{2}(t, x, y)
\end{array}\right) \geq\left(\begin{array}{l}
a_{11} x+b_{11} y-l_{1} \\
a_{12} x+b_{12} y-l_{2}
\end{array}\right), \quad \forall(t, x, y) \in[1, e] \times \mathbb{R}^{+} \times \mathbb{R}^{+} \text {. }
\end{aligned}
$$


(H4) There are $a_{2 i}, b_{2 i} \geq 0(i=1,2)$ and $r_{1}>0$ such that

$$
\begin{aligned}
& \left(\kappa_{2}+\kappa_{5} d_{h}\right) a_{21}+\kappa_{6} a_{22}<1, \quad\left(\kappa_{2}+d_{g} \kappa_{6}\right) b_{22}+\kappa_{5} b_{21}<1, \\
& \operatorname{det}\left(\begin{array}{cc}
1-\left(\kappa_{2}+\kappa_{5} d_{h}\right) a_{21}-\kappa_{6} a_{22} & -\left(\kappa_{2}+\kappa_{5} d_{h}\right) b_{21}-\kappa_{6} b_{22} \\
-\left(\kappa_{2}+d_{g} \kappa_{6}\right) a_{22}-\kappa_{5} a_{21} & 1-\left(\kappa_{2}+d_{g} \kappa_{6}\right) b_{22}-\kappa_{5} b_{21}
\end{array}\right)>0, \\
& \left(\begin{array}{l}
f_{1}(t, x, y) \\
f_{2}(t, x, y)
\end{array}\right) \leq\left(\begin{array}{l}
a_{21} x+b_{21} y \\
a_{22} x+b_{22} y
\end{array}\right), \quad \forall(t, x, y) \in[1, e] \times\left[0, r_{1}\right] \times\left[0, r_{1}\right] .
\end{aligned}
$$

(H5) There are $a_{3 i}, b_{3 i} \geq 0(i=1,2)$ and $r_{2}>0$ such that

$$
\begin{aligned}
& a_{31}\left(\kappa_{1}+\kappa_{3} d_{h}\right)+a_{32} \kappa_{4}<1, \quad b_{32}\left(\kappa_{1}+d_{g} \kappa_{4}\right)+b_{31} \kappa_{3}<1, \\
& \operatorname{det}\left(\begin{array}{cc}
b_{31}\left(\kappa_{1}+\kappa_{3} d_{h}\right)+b_{32} \kappa_{4} & a_{31}\left(\kappa_{1}+\kappa_{3} d_{h}\right)+a_{32} \kappa_{4}-1 \\
b_{32}\left(\kappa_{1}+d_{g} \kappa_{4}\right)+b_{31} \kappa_{3}-1 & a_{32}\left(\kappa_{1}+d_{g} \kappa_{4}\right)+a_{31} \kappa_{3}
\end{array}\right)>0, \\
& \left(\begin{array}{l}
f_{1}(t, x, y) \\
f_{2}(t, x, y)
\end{array}\right) \geq\left(\begin{array}{l}
a_{31} x+b_{31} y \\
a_{32} x+b_{32} y
\end{array}\right), \quad \forall(t, x, y) \in[1, e] \times\left[0, r_{2}\right] \times\left[0, r_{2}\right] .
\end{aligned}
$$

(H6) There are $a_{4 i}, b_{4 i} \geq 0(i=1,2)$ and $l_{3}, l_{4}>0$ such that

$$
\begin{aligned}
& \left(\kappa_{2}+\kappa_{5} d_{h}\right) a_{41}+\kappa_{6} a_{42}<1, \quad\left(\kappa_{2}+d_{g} \kappa_{6}\right) b_{42}+\kappa_{5} b_{41}<1, \\
& \operatorname{det}\left(\begin{array}{cc}
1-\left(\kappa_{2}+\kappa_{5} d_{h}\right) a_{41}-\kappa_{6} a_{42} & -\left(\kappa_{2}+\kappa_{5} d_{h}\right) b_{41}-\kappa_{6} b_{42} \\
-\left(\kappa_{2}+d_{g} \kappa_{6}\right) a_{42}-\kappa_{5} a_{41} & 1-\left(\kappa_{2}+d_{g} \kappa_{6}\right) b_{42}-\kappa_{5} b_{41}
\end{array}\right)>0 \\
& \left(\begin{array}{l}
f_{1}(t, x, y) \\
f_{2}(t, x, y)
\end{array}\right) \leq\left(\begin{array}{l}
a_{41} x+b_{41} y+l_{3} \\
a_{42} x+b_{42} y+l_{4}
\end{array}\right), \quad \forall(t, x, y) \in[1, e] \times \mathbb{R}^{+} \times \mathbb{R}^{+} .
\end{aligned}
$$

Let $B_{\rho}:=\{u \in E:\|u\|<\rho\}$ for $\rho>0$ in the sequel.

Theorem 3.1 Suppose that (H1)-(H4) hold. Then (1.1) has a positive solution.

Proof Let $S_{1}=\left\{(u, v) \in P \times P:(u, v)=A(u, v)+\lambda\left(\varphi_{1}, \varphi_{1}\right), \forall \lambda \geq 0\right\}$, where $\varphi_{1}$ is a fixed element in $P_{0}$. We claim that $S_{1}$ is a bounded set in $P \times P$. Note if there exists $(u, v) \in S_{1}$ such that

$$
u(t)=A_{1}(u, v)(t)+\lambda \varphi_{1}(t), \quad v(t)=A_{2}(u, v)(t)+\lambda \varphi_{1}(t) \quad \text { for } t \in[1, e]
$$

then this, together with Lemma 2.6, implies that

$$
u, v \in P_{0} \text {. }
$$

From (3.1) we have

$$
u(t) \geq A_{1}(u, v)(t), \quad v(t) \geq A_{2}(u, v)(t) \quad \text { for } t \in[1, e] .
$$


From the definitions of $A_{i}(i=1,2)$, multiplying by $\mu(t)$ and integrating from 1 to $e$, Lemmas 2.4 and 2.5 enable us to obtain

$$
\begin{aligned}
& \left(\begin{array}{l}
\int_{1}^{e} u(t) \mu(t) \frac{d t}{t} \\
\int_{1}^{e} v(t) \mu(t) \frac{d t}{t}
\end{array}\right) \\
& \quad\left(\begin{array}{c}
\int_{1}^{e} \mu(t)\left(\int_{1}^{e} G_{1}(t, s) f_{1}(s, u(s), v(s)) \frac{d s}{s}\right. \\
\quad+\frac{d_{h}(\log t)^{\beta-1}}{d_{g, h} \Gamma(\beta)} \int_{1}^{e} \int_{1}^{e} g(t) G_{1}(t, s) \frac{d t}{t} f_{1}(s, u(s), v(s)) \frac{d s}{s} \\
\left.\quad+\frac{(\log t)^{\beta-1}}{d_{g, h} \Gamma(\beta)} \int_{1}^{e} \int_{1}^{e} h(t) G_{1}(t, s) \frac{d t}{t} f_{2}(s, u(s), v(s)) \frac{d s}{s}\right) \frac{d t}{t} \\
\int_{1}^{e} \mu(t)\left(\int_{1}^{e} G_{1}(t, s) f_{2}(s, u(s), v(s)) \frac{d s}{s}\right. \\
\quad+\frac{d_{g}(\log t)^{\beta-1}}{d_{g, h} \Gamma(\beta)} \int_{1}^{e} \int_{1}^{e} h(t) G_{1}(t, s) \frac{d t}{t} f_{2}(s, u(s), v(s)) \frac{d s}{s} \\
\left.\quad+\frac{\left(\log t^{\beta-1}\right.}{d_{g, h} \Gamma(\beta)} \int_{1}^{e} \int_{1}^{e} g(t) G_{1}(t, s) \frac{d t}{t} f_{1}(s, u(s), v(s)) \frac{d s}{s}\right) \frac{d t}{t}
\end{array}\right) \\
& \geq\left(\begin{array}{c}
\left(\kappa_{1}+\kappa_{3} d_{h}\right) \int_{1}^{e} \mu(t) f_{1}(t, u(t), v(t)) \frac{d t}{t}+\kappa_{4} \int_{1}^{e} \mu(t) f_{2}(t, u(t), v(t)) \frac{d t}{t} \\
\left(\kappa_{1}+d_{g} \kappa_{4}\right) \int_{1}^{e} \mu(t) f_{2}(t, u(t), v(t)) \frac{d t}{t}+\kappa_{3} \int_{1}^{e} \mu(t) f_{1}(t, u(t), v(t)) \frac{d t}{t}
\end{array}\right)
\end{aligned}
$$

Combining this with (H3), we have

$$
\begin{gathered}
\left(\begin{array}{c}
\int_{1}^{e} u(t) \mu(t) \frac{d t}{t} \\
\int_{1}^{e} v(t) \mu(t) \frac{d t}{t}
\end{array}\right) \\
\geq\left(\begin{array}{c}
\left(\kappa_{1}+\kappa_{3} d_{h}\right) \int_{1}^{e} \mu(t)\left(a_{11} u(t)+b_{11} v(t)-l_{1}\right) \frac{d t}{t} \\
+\kappa_{4} \int_{1}^{e} \mu(t)\left(a_{12} u(t)+b_{12} v(t)-l_{2}\right) \frac{d t}{t} \\
\left(\kappa_{1}+d_{g} \kappa_{4}\right) \int_{1}^{e} \mu(t)\left(a_{12} u(t)+b_{12} v(t)-l_{2}\right) \frac{d t}{t} \\
+\kappa_{3} \int_{1}^{e} \mu(t)\left(a_{11} u(t)+b_{11} v(t)-l_{1}\right) \frac{d t}{t}
\end{array}\right),
\end{gathered}
$$

and

$$
\begin{gathered}
\left(\begin{array}{cc}
b_{11}\left(\kappa_{1}+\kappa_{3} d_{h}\right)+b_{12} \kappa_{4} & a_{11}\left(\kappa_{1}+\kappa_{3} d_{h}\right)+a_{12} \kappa_{4}-1 \\
b_{12}\left(\kappa_{1}+d_{g} \kappa_{4}\right)+b_{11} \kappa_{3}-1 & a_{12}\left(\kappa_{1}+d_{g} \kappa_{4}\right)+a_{11} \kappa_{3}
\end{array}\right)\left(\begin{array}{l}
\int_{1}^{e} v(t) \mu(t) \frac{d t}{t} \\
\int_{1}^{e} u(t) \mu(t) \frac{d t}{t}
\end{array}\right) \\
\leq\left(\begin{array}{l}
\left(\left(\kappa_{1}+\kappa_{3} d_{h}\right) l_{1}+\kappa_{4} l_{2}\right) \int_{1}^{e} \mu(t) \frac{d t}{t} \\
\left(\left(\kappa_{1}+d_{g} \kappa_{4}\right) l_{2}+\kappa_{3} l_{1}\right) \int_{1}^{e} \mu(t) \frac{d t}{t}
\end{array}\right)=\left(\begin{array}{l}
\frac{\left(\kappa_{1}+\kappa_{3} d_{h}\right) l_{1}+\kappa_{4} l_{2}}{\Gamma(\beta+2)} \\
\frac{\left(\kappa_{1}+d_{g} \kappa_{4} l_{2}+\kappa_{3} l_{1}\right.}{\Gamma(\beta+2)}
\end{array}\right) .
\end{gathered}
$$

Solving this matrix inequality, we have

$$
\left(\begin{array}{l}
\int_{1}^{e} v(t) \mu(t) \frac{d t}{t} \\
\int_{1}^{e} u(t) \mu(t) \frac{d t}{t}
\end{array}\right) \leq \frac{\left(\begin{array}{cc}
a_{12}\left(\kappa_{1}+d_{g} \kappa_{4}\right)+a_{11} \kappa_{3} & 1-a_{11}\left(\kappa_{1}+\kappa_{3} d_{h}\right)+a_{12} \kappa_{4} \\
1-b_{12}\left(\kappa_{1}+d_{g} \kappa_{4}\right)+b_{11} \kappa_{3} & b_{11}\left(\kappa_{1}+\kappa_{3} d_{h}\right)+b_{12} \kappa_{4}
\end{array}\right)}{\operatorname{det}\left(\begin{array}{cc}
b_{11}\left(\kappa_{1}+\kappa_{3} d_{h}\right)+b_{12} \kappa_{4} & a_{11}\left(\kappa_{1}+\kappa_{3} d_{h}\right)+a_{12} \kappa_{4}-1 \\
b_{12}\left(\kappa_{1}+d_{g} \kappa_{4}\right)+b_{11} \kappa_{3}-1 & a_{12}\left(\kappa_{1}+d_{g} \kappa_{4}\right)+a_{11} \kappa_{3}
\end{array}\right)}\left(\begin{array}{c}
\frac{\left(\kappa_{1}+\kappa_{3} d_{h}\right) l_{1}+\kappa_{4} l_{2}}{\Gamma(\beta+2)} \\
\frac{\left(\kappa_{1}+d_{g} \kappa_{4}\right) l_{2}+\kappa_{3} l_{1}}{\Gamma(\beta+2)}
\end{array}\right) .
$$

Hence, there exist $M_{1}>0, M_{2}>0$ such that

$$
\left(\begin{array}{l}
\int_{1}^{e} v(t) \mu(t) \frac{d t}{t} \\
\int_{1}^{e} u(t) \mu(t) \frac{d t}{t}
\end{array}\right) \leq\left(\begin{array}{l}
M_{1} \\
M_{2}
\end{array}\right)
$$

Note (3.2), and we find

$$
\left(\begin{array}{l}
\|v\| \\
\|u\|
\end{array}\right) \leq\left(\begin{array}{l}
\frac{M_{1}(\beta-1) \Gamma(2 \beta+2)}{\beta^{2} \Gamma(\beta)} \\
\frac{M_{2}(\beta-1) \Gamma(2 \beta+2)}{\beta^{2} \Gamma(\beta)}
\end{array}\right) .
$$


This proves that $S_{1}$ is bounded in $P \times P$. As a result, if we choose $R_{1}>\left\{r_{1}, \frac{M_{1}(\beta-1) \Gamma(2 \beta+2)}{\beta^{2} \Gamma(\beta)}\right.$, $\left.\frac{M_{2}(\beta-1) \Gamma(2 \beta+2)}{\beta^{2} \Gamma(\beta)}\right\}\left(r_{1}\right.$ is defined by $\left.(\mathrm{H} 4)\right)$, then we have

$$
(u, v) \neq A(u, v)+\lambda\left(\varphi_{1}, \varphi_{1}\right), \quad \text { for }(u, v) \in \partial B_{R_{1}} \cap(P \times P), \forall \lambda \geq 0 .
$$

From Lemma 2.7 we have

$$
i\left(A, B_{R_{1}} \cap(P \times P), P \times P\right)=0 .
$$

Next we claim that

$$
(u, v) \neq \lambda A(u, v), \quad \text { for }(u, v) \in \partial B_{r_{1}} \cap(P \times P), \forall \lambda \in[0,1],
$$

where $r_{1}$ is defined by (H4). Suppose (3.7) is not true. Then there exist $(u, v) \in \partial B_{r_{1}} \cap(P \times P)$ and $\lambda \in[0,1]$ such that $(u, v)=\lambda A(u, v)$, which implies that

$$
u(t) \leq A_{1}(u, v)(t), \quad v(t) \leq A_{2}(u, v)(t) \quad \text { for } t \in[1, e] .
$$

Multiplying by $\mu(t)$ and integrating from 1 to $e$, Lemmas 2.4 and 2.5 enable us to obtain

$$
\begin{aligned}
& \left(\begin{array}{l}
\int_{1}^{e} u(t) \mu(t) \frac{d t}{t} \\
\int_{1}^{e} v(t) \mu(t) \frac{d t}{t}
\end{array}\right) \\
& \quad\left(\begin{array}{c}
\int_{1}^{e} \mu(t)\left(\int_{1}^{e} G_{1}(t, s) f_{1}(s, u(s), v(s)) \frac{d s}{s}\right. \\
\quad+\frac{d_{h}(\log t)^{\beta-1}}{d_{g, h} \Gamma(\beta)} \int_{1}^{e} \int_{1}^{e} g(t) G_{1}(t, s) \frac{d t}{t} f_{1}(s, u(s), v(s)) \frac{d s}{s} \\
\left.\quad+\frac{(\log t)^{\beta-1}}{d_{g, h} \Gamma(\beta)} \int_{1}^{e} \int_{1}^{e} h(t) G_{1}(t, s) \frac{d t}{t} f_{2}(s, u(s), v(s)) \frac{d s}{s}\right) \frac{d t}{t} \\
\int_{1}^{e} \mu(t)\left(\int_{1}^{e} G_{1}(t, s) f_{2}(s, u(s), v(s)) \frac{d s}{s}\right. \\
\quad+\frac{d_{g}(\log t)^{\beta-1}}{d_{g, h} \Gamma(\beta)} \int_{1}^{e} \int_{1}^{e} h(t) G_{1}(t, s) \frac{d t}{t} f_{2}(s, u(s), v(s)) \frac{d s}{s} \\
\left.\quad+\frac{\left(\log t^{\beta-1}\right.}{d_{g, h} \Gamma(\beta)} \int_{1}^{e} \int_{1}^{e} g(t) G_{1}(t, s) \frac{d t}{t} f_{1}(s, u(s), v(s)) \frac{d s}{s}\right) \frac{d t}{t}
\end{array}\right) \\
& \leq\left(\begin{array}{c}
\left(\kappa_{2}+\kappa_{5} d_{h}\right) \int_{1}^{e} \mu(t) f_{1}(t, u(t), v(t)) \frac{d t}{t}+\kappa_{6} \int_{1}^{e} \mu(t) f_{2}(t, u(t), v(t)) \frac{d t}{t} \\
\left(\kappa_{2}+d_{g} \kappa_{6}\right) \int_{1}^{e} \mu(t) f_{2}(t, u(t), v(t)) \frac{d t}{t}+\kappa_{5} \int_{1}^{e} \mu(t) f_{1}(t, u(t), v(t)) \frac{d t}{t}
\end{array}\right) .
\end{aligned}
$$

Substituting (H4) into this matrix inequality, we obtain

$$
\begin{aligned}
& \left(\begin{array}{l}
\int_{1}^{e} u(t) \mu(t) \frac{d t}{t} \\
\int_{1}^{e} v(t) \mu(t) \frac{d t}{t}
\end{array}\right) \\
& \quad \leq\left(\begin{array}{l}
\left(\kappa_{2}+\kappa_{5} d_{h}\right) \int_{1}^{e} \mu(t)\left(a_{21} u(t)+b_{21} v(t)\right) \frac{d t}{t}+\kappa_{6} \int_{1}^{e} \mu(t)\left(a_{22} u(t)+b_{22} v(t)\right) \frac{d t}{t} \\
\left(\kappa_{2}+d_{g} \kappa_{6}\right) \int_{1}^{e} \mu(t)\left(a_{22} u(t)+b_{22} v(t)\right) \frac{d t}{t}+\kappa_{5} \int_{1}^{e} \mu(t)\left(a_{21} u(t)+b_{21} v(t)\right) \frac{d t}{t}
\end{array}\right) .
\end{aligned}
$$

Consequently, we get

$$
\left(\begin{array}{cc}
1-\left(\kappa_{2}+\kappa_{5} d_{h}\right) a_{21}-\kappa_{6} a_{22} & -\left(\kappa_{2}+\kappa_{5} d_{h}\right) b_{21}-\kappa_{6} b_{22} \\
-\left(\kappa_{2}+d_{g} \kappa_{6}\right) a_{22}-\kappa_{5} a_{21} & 1-\left(\kappa_{2}+d_{g} \kappa_{6}\right) b_{22}-\kappa_{5} b_{21}
\end{array}\right)\left(\begin{array}{c}
\int_{1}^{e} u(t) \mu(t) \frac{d t}{t} \\
\int_{1}^{e} v(t) \mu(t) \frac{d t}{t}
\end{array}\right) \leq\left(\begin{array}{l}
0 \\
0
\end{array}\right) .
$$


Therefore, (H4) implies that

$$
\left(\begin{array}{l}
\int_{1}^{e} u(t) \mu(t) \frac{d t}{t} \\
\int_{1}^{e} v(t) \mu(t) \frac{d t}{t}
\end{array}\right) \leq \frac{\left(\begin{array}{cc}
1-\left(\kappa_{2}+d_{g} \kappa_{6}\right) b_{22}-\kappa_{5} b_{21} & \left(\kappa_{2}+\kappa_{5} d_{h}\right) b_{21}+\kappa_{6} b_{22} \\
\left(\kappa_{2}+d_{g} \kappa_{6}\right) a_{22}+\kappa_{5} a_{21} & 1-\left(\kappa_{2}+\kappa_{5} d_{h}\right) a_{21}-\kappa_{6} a_{22}
\end{array}\right)\left(\begin{array}{l}
0 \\
0
\end{array}\right)}{\operatorname{det}\left(\begin{array}{cc}
1-\left(\kappa_{2}+\kappa_{5} d_{h}\right) a_{21}-\kappa_{6} a_{22} & -\left(\kappa_{2}+\kappa_{5} d_{h}\right) b_{21}-\kappa_{6} b_{22} \\
-\left(\kappa_{2}+d_{g} \kappa_{6}\right) a_{22}-\kappa_{5} a_{21} & 1-\left(\kappa_{2}+d_{g} \kappa_{6}\right) b_{22}-\kappa_{5} b_{21}
\end{array}\right)}=\left(\begin{array}{l}
0 \\
0
\end{array}\right) .
$$

Hence,

$$
\int_{1}^{e} u(t) \mu(t) \frac{d t}{t}=0, \quad \int_{1}^{e} v(t) \mu(t) \frac{d t}{t}=0
$$

Note that $\mu(t) \not \equiv 0$ for $t \in[1, e]$, so $u(t)=v(t) \equiv 0, t \in[1, e]$, which implies that $\|u\|=\|v\|=$ 0 , contradicting $(u, v) \in \partial B_{r_{1}} \cap(P \times P)$. As a result, (3.7) holds. From Lemma 2.8 we have

$$
i\left(A, B_{r_{1}} \cap(P \times P), P \times P\right)=1 .
$$

From (3.6) and (3.11) we have

$$
\begin{aligned}
& i\left(A,\left(B_{R_{1}} \backslash \bar{B}_{r_{1}}\right) \cap(P \times P), P \times P\right) \\
& \quad=i\left(A, B_{R_{1}} \cap(P \times P), P \times P\right)-i\left(A, B_{r_{1}} \cap(P \times P), P \times P\right)=0-1=-1 .
\end{aligned}
$$

Therefore the operator $A$ has at least one fixed point on $\left(B_{R_{1}} \backslash \bar{B}_{r_{1}}\right) \cap(P \times P)$. Equivalently, (1.1) has at least one positive solution. This completes the proof.

Theorem 3.2 Suppose that (H1)-(H2), (H5)-(H6) hold. Then (1.1) has a positive solution.

Proof We use similar methods as in Theorem 3.1 to prove this theorem. We first claim that

$$
(u, v) \neq A(u, v)+\lambda\left(\varphi_{2}, \varphi_{2}\right), \quad \text { for }(u, v) \in \partial B_{r_{2}} \cap(P \times P), \forall \lambda \geq 0,
$$

where $\varphi_{2} \in P$ is a given element. Suppose the claim is not true. Then there exist $(u, v) \in$ $\partial B_{r_{2}} \cap(P \times P)$ and $\lambda \geq 0$ such that $(u, v)=A(u, v)+\lambda\left(\varphi_{2}, \varphi_{2}\right)$, which implies that

$$
u(t) \geq A_{1}(u, v)(t), \quad v(t) \geq A_{2}(u, v)(t) \quad \text { for } t \in[1, e] .
$$

Similar to (3.4), (3.5), from (H5) we obtain

$$
\begin{aligned}
& \left(\begin{array}{l}
\int_{1}^{e} u(t) \mu(t) \frac{d t}{t} \\
\int_{1}^{e} v(t) \mu(t) \frac{d t}{t}
\end{array}\right) \\
& \geq\left(\begin{array}{l}
\left(\kappa_{1}+\kappa_{3} d_{h}\right) \int_{1}^{e} \mu(t)\left(a_{31} u(t)+b_{31} v(t)\right) \frac{d t}{t}+\kappa_{4} \int_{1}^{e} \mu(t)\left(a_{32} u(t)+b_{32} v(t)\right) \frac{d t}{t} \\
\left(\kappa_{1}+d_{g} \kappa_{4}\right) \int_{1}^{e} \mu(t)\left(a_{32} u(t)+b_{32} v(t)\right) \frac{d t}{t}+\kappa_{3} \int_{1}^{e} \mu(t)\left(a_{31} u(t)+b_{31} v(t)\right) \frac{d t}{t}
\end{array}\right)
\end{aligned}
$$

and

$$
\left(\begin{array}{cc}
b_{31}\left(\kappa_{1}+\kappa_{3} d_{h}\right)+b_{32} \kappa_{4} & a_{31}\left(\kappa_{1}+\kappa_{3} d_{h}\right)+a_{32} \kappa_{4}-1 \\
b_{32}\left(\kappa_{1}+d_{g} \kappa_{4}\right)+b_{31} \kappa_{3}-1 & a_{32}\left(\kappa_{1}+d_{g} \kappa_{4}\right)+a_{31} \kappa_{3}
\end{array}\right)\left(\begin{array}{l}
\int_{1}^{e} v(t) \mu(t) \frac{d t}{t} \\
\int_{1}^{e} u(t) \mu(t) \frac{d t}{t}
\end{array}\right) \leq\left(\begin{array}{l}
0 \\
0
\end{array}\right)
$$


Thus $u(t)=v(t) \equiv 0$ for $t \in[1, e]$, and $\|u\|=\|v\|=0$, which contradicts $(u, v) \in \partial B_{r_{2}} \cap$ $(P \times P)$. Consequently, (3.12) holds, and from Lemma 2.7 we have

$$
i\left(A, B_{r_{2}} \cap(P \times P), P \times P\right)=0 .
$$

Let $S_{2}=\{(u, v) \in P \times P:(u, v)=\lambda A(u, v), \forall \lambda \in[0,1]\}$. Now we prove that $S_{2}$ is bounded in $P \times P$. Note if there exists $(u, v) \in S_{2}$, then

$$
u(t) \leq A_{1}(u, v)(t), \quad v(t) \leq A_{2}(u, v)(t) \quad \text { for } t \in[1, e],
$$

and similar to (3.9), (3.10), and by (H6) we have

$$
\begin{aligned}
& \left(\begin{array}{l}
\int_{1}^{e} u(t) \mu(t) \frac{d t}{t} \\
\int_{1}^{e} v(t) \mu(t) \frac{d t}{t}
\end{array}\right) \\
& \leq\left(\begin{array}{c}
\left(\kappa_{2}+\kappa_{5} d_{h}\right) \int_{1}^{e} \mu(t)\left(a_{41} u(t)+b_{41} v(t)+l_{3}\right) \frac{d t}{t} \\
+\kappa_{6} \int_{1}^{e} \mu(t)\left(a_{42} u(t)+b_{42} v(t)+l_{4}\right) \frac{d t}{t} \\
\left(\kappa_{2}+d_{g} \kappa_{6}\right) \int_{1}^{e} \mu(t)\left(a_{42} u(t)+b_{42} v(t)+l_{4}\right) \frac{d t}{t} \\
\left.+\kappa_{5} \int_{1}^{e} \mu(t)\left(a_{41} u(t)+b_{41} v(t)+l_{3}\right)\right) \frac{d t}{t}
\end{array}\right) .
\end{aligned}
$$

Thus

$$
\begin{aligned}
& \left(\begin{array}{cc}
1-\left(\kappa_{2}+\kappa_{5} d_{h}\right) a_{41}-\kappa_{6} a_{42} & -\left(\kappa_{2}+\kappa_{5} d_{h}\right) b_{41}-\kappa_{6} b_{42} \\
-\left(\kappa_{2}+d_{g} \kappa_{6}\right) a_{42}-\kappa_{5} a_{41} & 1-\left(\kappa_{2}+d_{g} \kappa_{6}\right) b_{42}-\kappa_{5} b_{41}
\end{array}\right)\left(\begin{array}{l}
\int_{1}^{e} u(t) \mu(t) \frac{d t}{t} \\
\int_{1}^{e} v(t) \mu(t) \frac{d t}{t}
\end{array}\right) \\
& \leq\left(\begin{array}{l}
\frac{\left(\kappa_{2}+\kappa_{5} d_{h}\right) l_{3}+\kappa_{6} l_{4}}{\Gamma(\beta+2)} \\
\frac{\left(\kappa_{2}+d_{g} \kappa_{6}\right) l_{4}+\kappa_{5} l_{3}}{\Gamma(\beta+2)}
\end{array}\right) .
\end{aligned}
$$

Solving this matrix inequality, we have

$$
\left(\begin{array}{l}
\int_{1}^{e} u(t) \mu(t) \frac{d t}{t} \\
\int_{1}^{e} v(t) \mu(t) \frac{d t}{t}
\end{array}\right) \leq \frac{\left(\begin{array}{cc}
1-\left(\kappa_{2}+d_{g} \kappa_{6}\right) b_{42}-\kappa_{5} b_{41} & \left(\kappa_{2}+\kappa_{5} d_{h}\right) b_{41}+\kappa_{6} b_{42} \\
\left(\kappa_{2}+d_{g} \kappa_{6}\right) a_{42}+\kappa_{5} a_{41} & 1-\left(\kappa_{2}+\kappa_{5} d_{h}\right) a_{41}-\kappa_{6} a_{42}
\end{array}\right)}{\operatorname{det}\left(\begin{array}{cc}
1-\left(\kappa_{2}+\kappa_{5} d_{h}\right) a_{41}-\kappa_{6} a_{42} & -\left(\kappa_{2}+\kappa_{5} d_{h}\right) b_{41}-\kappa_{6} b_{42} \\
-\left(\kappa_{2}+d_{g} \kappa_{6}\right) a_{42}-\kappa_{5} a_{41} & 1-\left(\kappa_{2}+d_{g} \kappa_{6}\right) b_{42}-\kappa_{5} b_{41}
\end{array}\right)}\left(\begin{array}{c}
\frac{\left(\kappa_{2}+\kappa_{5} d_{h}\right) l_{3}+\kappa_{6} l_{4}}{\Gamma(\beta+2)} \\
\frac{\left(\kappa_{2}+d_{g} \kappa_{6}\right) l_{4}+\kappa_{5} l_{3}}{\Gamma(\beta+2)}
\end{array}\right) .
$$

Hence, there exist $M_{3}>0, M_{4}>0$ such that

$$
\left(\begin{array}{l}
\int_{1}^{e} u(t) \mu(t) \frac{d t}{t} \\
\int_{1}^{e} v(t) \mu(t) \frac{d t}{t}
\end{array}\right) \leq\left(\begin{array}{l}
M_{3} \\
M_{4}
\end{array}\right)
$$

Note that $(u, v) \in S_{2}$, and from Lemma 2.6, we find $u, v \in P_{0}$. Thus, we obtain

$$
\left(\begin{array}{l}
\|u\| \\
\|v\|
\end{array}\right) \leq\left(\begin{array}{l}
\frac{M_{3}(\beta-1) \Gamma(2 \beta+2)}{\beta^{2} \Gamma(\beta)} \\
\frac{M_{4}(\beta-1) \Gamma(2 \beta+2)}{\beta^{2} \Gamma(\beta)}
\end{array}\right)
$$

This proves that $S_{2}$ is bounded in $P \times P$. As a result, if we take $R_{2}>\left\{r_{2}, \frac{M_{3}(\beta-1) \Gamma(2 \beta+2)}{\beta^{2} \Gamma(\beta)}\right.$, $\left.\frac{M_{4}(\beta-1) \Gamma(2 \beta+2)}{\beta^{2} \Gamma(\beta)}\right\}\left(r_{2}\right.$ is defined by (H5)), we conclude that

$$
(u, v) \neq \lambda A(u, v), \quad \text { for }(u, v) \in \partial B_{R_{2}} \cap(P \times P), \forall \lambda \in[0,1] .
$$


From Lemma 2.8 we have

$$
i\left(A, B_{R_{2}} \cap(P \times P), P \times P\right)=1 \text {. }
$$

From (3.13) and (3.15) we have

$$
\begin{aligned}
& i\left(A,\left(B_{R_{2}} \backslash \bar{B}_{r_{2}}\right) \cap(P \times P), P \times P\right) \\
& \quad=i\left(A, B_{R_{2}} \cap(P \times P), P \times P\right)-i\left(A, B_{r_{2}} \cap(P \times P), P \times P\right)=1-0=1 .
\end{aligned}
$$

Therefore the operator $A$ has at least one fixed point on $\left(B_{R_{2}} \backslash \bar{B}_{r_{2}}\right) \cap(P \times P)$. Equivalently, (1.1) has at least one positive solution. This completes the proof.

Example 3.3 Let $\beta=2.5, h(t)=g(t)=\log t$ for $t \in[1, e]$. Then $d_{h}=d_{g}=\int_{1}^{e}(\log t)^{\beta} \frac{d t}{t}=\frac{2}{7}$, $d_{g, h}=1-\int_{1}^{e} h(t)(\log t)^{\beta-1} \frac{d t}{t} \cdot \int_{1}^{e} g(t)(\log t)^{\beta-1} \frac{d t}{t}=1-\frac{4}{49}=\frac{45}{49}$. This implies that (H2) holds. Next, we calculate $\kappa_{i}(i=1,2,3,4,5,6)$ as follows:

$$
\begin{aligned}
\kappa_{1} & =\frac{\beta^{2} \Gamma(\beta)}{\Gamma(2 \beta+2)}=\frac{2.5^{2} \Gamma(2.5)}{\Gamma(7)} \approx 0.01154 \\
\kappa_{2} & =\frac{\beta-1}{\Gamma(\beta+2)}=\frac{1.5}{\Gamma(4.5)} \approx 0.129, \\
\kappa_{3} & =\kappa_{4}=\frac{\beta}{d_{g, h} \Gamma(2 \beta+1)} \int_{1}^{e}(\log t)(\log t)^{\beta-1}(1-\log t) \frac{d t}{t} \\
& =\frac{2.5}{\frac{45}{49} \Gamma(6)} \int_{1}^{e}(\log t)^{2.5}(1-\log t) \frac{d t}{t} \approx 0.00144, \\
\kappa_{5} & =\kappa_{6}=\frac{\beta(\beta-1)}{d_{g, h} \Gamma(2 \beta+1)} \int_{1}^{e}(\log t) \frac{d t}{t}=\frac{2.5 \times 1.5}{\frac{45}{49} \Gamma(6)} \int_{1}^{e}(\log t) \frac{d t}{t} \approx 0.017 .
\end{aligned}
$$

Case 1. Let $a_{11}=10, a_{12}=600, b_{11}=630, b_{12}=7, a_{21}=3, a_{22}=4, b_{21}=3, b_{22}=2$. Then we have

$$
\begin{aligned}
& a_{11}\left(\kappa_{1}+\kappa_{3} d_{h}\right)+a_{12} \kappa_{4}=10 \times 0.012+600 \times 0.00144<1, \\
& b_{12}\left(\kappa_{1}+d_{g} \kappa_{4}\right)+b_{11} \kappa_{3}=7 \times 0.012+630 \times 0.00144<1, \\
& \left(\kappa_{2}+\kappa_{5} d_{h}\right) a_{21}+\kappa_{6} a_{22}=0.134 \times 3+0.017 \times 4<1, \\
& \left(\kappa_{2}+d_{g} \kappa_{6}\right) b_{22}+\kappa_{5} b_{21}=0.134 \times 2+0.017 \times 3<1, \\
& \left|\begin{array}{cc}
b_{11}\left(\kappa_{1}+\kappa_{3} d_{h}\right)+b_{12} \kappa_{4} & a_{11}\left(\kappa_{1}+\kappa_{3} d_{h}\right)+a_{12} \kappa_{4}-1 \\
b_{12}\left(\kappa_{1}+d_{g} \kappa_{4}\right)+b_{11} \kappa_{3}-1 & a_{12}\left(\kappa_{1}+d_{g} \kappa_{4}\right)+a_{11} \kappa_{3}
\end{array}\right|=\left|\begin{array}{cc}
7.57 & -0.016 \\
-0.009 & 7.21
\end{array}\right|>0,
\end{aligned}
$$

and

$$
\left|\begin{array}{cc}
1-\left(\kappa_{2}+\kappa_{5} d_{h}\right) a_{21}-\kappa_{6} a_{22} & -\left(\kappa_{2}+\kappa_{5} d_{h}\right) b_{21}-\kappa_{6} b_{22} \\
-\left(\kappa_{2}+d_{g} \kappa_{6}\right) a_{22}-\kappa_{5} a_{21} & 1-\left(\kappa_{2}+d_{g} \kappa_{6}\right) b_{22}-\kappa_{5} b_{21}
\end{array}\right|=\left|\begin{array}{cc}
0.53 & -0.436 \\
-0.587 & 0.681
\end{array}\right|>0 .
$$


Let $f_{1}(t, x, y)=(10 x+630 y)^{\gamma_{1}}, f_{2}(t, x, y)=(600 x+7 y)^{\gamma_{2}}$ for $t \in[1, e], x, y \in \mathbb{R}^{+}, \gamma_{1}, \gamma_{2}>1$. Then we have

$$
\begin{aligned}
& \liminf _{a_{11} x+b_{11} y \rightarrow+\infty} \frac{f_{1}(t, x, y)}{a_{11} x+b_{11} y}=\liminf _{10 x+630 y \rightarrow+\infty} \frac{(10 x+630 y)^{\gamma_{1}}}{10 x+630 y}=+\infty, \\
& \text { uniformly on } t \in[1, e], \\
& \liminf _{a_{12} x+b_{12} y \rightarrow+\infty} \frac{f_{2}(t, x, y)}{a_{12} x+b_{12} y}=\liminf _{600 x+7 y \rightarrow+\infty} \frac{(600 x+7 y)^{\gamma_{2}}}{600 x+7 y}=+\infty, \quad \text { uniformly on } t \in[1, e], \\
& \limsup _{a_{21} x+b_{21} y \rightarrow 0^{+}} \frac{f_{1}(t, x, y)}{a_{21} x+b_{21} y}=\limsup _{3 x+3 y \rightarrow 0^{+}} \frac{(10 x+630 y)^{\gamma_{1}}}{3 x+3 y}=0, \quad \text { uniformly on } t \in[1, e],
\end{aligned}
$$

and

$$
\limsup _{a_{22} x+b_{22} y \rightarrow 0^{+}} \frac{f_{2}(t, x, y)}{a_{22} x+b_{22} y}=\limsup _{4 x+2 y \rightarrow 0^{+}} \frac{(600 x+7 y)^{\gamma_{2}}}{4 x+2 y}=0, \quad \text { uniformly on } t \in[1, e] .
$$

As a result, (H3)-(H4) hold.

Case 2. Let $a_{31}=8, a_{32}=620, b_{31}=630, b_{32}=7, a_{41}=3, a_{42}=4, b_{41}=3, b_{42}=2$. Then we have

$$
\begin{aligned}
& a_{31}\left(\kappa_{1}+\kappa_{3} d_{h}\right)+a_{32} \kappa_{4}=8 \times 0.012+620 \times 0.00144<1, \\
& b_{32}\left(\kappa_{1}+d_{g} \kappa_{4}\right)+b_{31} \kappa_{3}=7 \times 0.012+630 \times 0.00144<1, \\
& \left(\kappa_{2}+\kappa_{5} d_{h}\right) a_{41}+\kappa_{6} a_{42}=0.134 \times 3+0.017 \times 4<1, \\
& \left(\kappa_{2}+d_{g} \kappa_{6}\right) b_{42}+\kappa_{5} b_{41}=0.134 \times 2+0.017 \times 3<1, \\
& \left|\begin{array}{cc}
b_{31}\left(\kappa_{1}+\kappa_{3} d_{h}\right)+b_{32} \kappa_{4} & a_{31}\left(\kappa_{1}+\kappa_{3} d_{h}\right)+a_{32} \kappa_{4}-1 \\
b_{32}\left(\kappa_{1}+d_{g} \kappa_{4}\right)+b_{31} \kappa_{3}-1 & a_{32}\left(\kappa_{1}+d_{g} \kappa_{4}\right)+a_{31} \kappa_{3}
\end{array}\right|=\left|\begin{array}{cc}
7.57 & -0.0112 \\
-0.009 & 7.45
\end{array}\right|>0,
\end{aligned}
$$

and

$$
\left|\begin{array}{cc}
1-\left(\kappa_{2}+\kappa_{5} d_{h}\right) a_{41}-\kappa_{6} a_{42} & -\left(\kappa_{2}+\kappa_{5} d_{h}\right) b_{41}-\kappa_{6} b_{42} \\
-\left(\kappa_{2}+d_{g} \kappa_{6}\right) a_{42}-\kappa_{5} a_{41} & 1-\left(\kappa_{2}+d_{g} \kappa_{6}\right) b_{42}-\kappa_{5} b_{41}
\end{array}\right|=\left|\begin{array}{cc}
0.53 & -0.436 \\
-0.587 & 0.681
\end{array}\right|>0 .
$$

Let $f_{1}(t, x, y)=(8 x+630 y)^{\gamma_{3}}, f_{2}(t, x, y)=(620 x+7 y)^{\gamma_{4}}$ for $t \in[1, e], x, y \in \mathbb{R}^{+}, \gamma_{3}, \gamma_{4} \in(0,1)$.

Then we have

$$
\begin{aligned}
& \liminf _{a_{31} x+b_{31} y \rightarrow 0^{+}} \frac{f_{1}(t, x, y)}{a_{31} x+b_{31} y}=\liminf _{8 x+630 y \rightarrow 0^{+}} \frac{(8 x+630 y)^{\gamma_{3}}}{8 x+630 y}=+\infty, \quad \text { uniformly on } t \in[1, e], \\
& \liminf _{a_{32} x+b_{32} y \rightarrow 0^{+}} \frac{f_{2}(t, x, y)}{a_{32} x+b_{32} y}=\liminf _{620 x+7 y \rightarrow 0^{+}} \frac{(620 x+7 y)^{\gamma_{4}}}{620 x+7 y}=+\infty, \quad \text { uniformly on } t \in[1, e], \\
& \limsup _{a_{41} x+b_{41} y \rightarrow+\infty} \frac{f_{1}(t, x, y)}{a_{41} x+b_{41} y}=\limsup _{3 x+3 y \rightarrow+\infty} \frac{(8 x+630 y)^{\gamma_{3}}}{3 x+3 y}=0, \quad \text { uniformly on } t \in[1, e],
\end{aligned}
$$

and

$$
\limsup _{a_{42} x+b_{42} y \rightarrow+\infty} \frac{f_{2}(t, x, y)}{a_{42} x+b_{42} y}=\limsup _{4 x+2 y \rightarrow+\infty} \frac{(620 x+7 y)^{\gamma_{4}}}{4 x+2 y}=0, \quad \text { uniformly on } t \in[1, e] .
$$

As a result, (H5)-(H6) hold. 


\section{Acknowledgements}

Not applicable.

\section{Funding}

This work was supported financially by the National Natural Science Foundation of China (11871302, 11601048), Natural Science Foundation of Chongaing (cstc2016jcyjA0181), Doctoral Scientific Research Foundation of Qufu Normal University and Youth Foundation of Qufu Normal University (BSQD20130140), and Natural Science Foundation of Chongqing Normal University (16XYY24).

\section{Availability of data and materials}

Not applicable.

\section{Competing interests}

The authors declare that there is no conflict of interests regarding the publication of this paper

\section{Authors' contributions}

All authors contributed equally and significantly in writing this article. All authors read and approved the final manuscript.

\section{Author details}

${ }^{1}$ School of Mathematical Sciences, Qufu Normal University, Qufu, China. ${ }^{2}$ School of Mathematics, Statistics and Applied Mathematics, National University of Ireland, Galway, Ireland. ${ }^{3}$ School of Mathematical Sciences, Chongqing Normal University, Chongqing, China. ${ }^{4}$ College of Mathematics and System Sciences, Shandong University of Science and Technology, Qingdao, China.

\section{Publisher's Note}

Springer Nature remains neutral with regard to jurisdictional claims in published maps and institutional affiliations.

Received: 26 March 2019 Accepted: 14 July 2019 Published online: 23 July 2019

\section{References}

1. Zhang, X., Wu, J., Liu, L., Wu, Y., Cui, Y.: Convergence analysis of iterative scheme and error estimation of positive solution for a fractional differential equation. Math. Model. Anal. 23(4), 611-626 (2018)

2. Wu, J., Zhang, X., Liu, L., Wu, Y., Cui, Y.: The convergence analysis and error estimation for unique solution of a p-Laplacian fractional differential equation with singular decreasing nonlinearity. Bound. Value Probl. 2018, Article ID $82(2018)$

3. He, J., Zhang, X., Liu, L., Wu, Y., Cui, Y.: Existence and asymptotic analysis of positive solutions for a singular fractional differential equation with nonlocal boundary conditions. Bound. Value Probl. 2018, Article ID 189 (2018)

4. Cui, Y.: Uniqueness of solution for boundary value problems for fractional differential equations. Appl. Math. Lett. 51 48-54 (2016)

5. Cui, Y., Ma, W., Sun, Q., Su, X.: New uniqueness results for boundary value problem of fractional differential equation. Nonlinear Anal., Model. Control 23(1), 31-39 (2018)

6. Zou, Y., He, G.: On the uniqueness of solutions for a class of fractional differential equations. Appl. Math. Lett. 74 68-73 (2017)

7. Yue, Z., Zou, Y.: New uniqueness results for fractional differential equation with dependence on the first order derivative. Adv. Differ. Equ. 2019, Article ID 38 (2019)

8. Zhai, C., Li, P., Li, H.: Single upper-solution or lower-solution method for Langevin equations with two fractional orders. Adv. Differ. Equ. 2018, Article ID 360 (2018)

9. Ahmad, B., Alghanmi, M., Ntouyas, S.K., Alsaedi, A.: Fractional differential equations involving generalized derivative with Stieltjes and fractional integral boundary conditions. Appl. Math. Lett. 84,111-117 (2018)

10. Sun, Q., Meng, S., Cui, Y.: Existence results for fractional order differential equation with nonlocal Erdélyi-Kober and generalized Riemann-Liouville type integral boundary conditions at resonance. Adv. Differ. Equ. 2018, Article ID 243 (2018)

11. Sheng, K., Zhang, W., Bai, Z.: Positive solutions to fractional boundary-value problems with $p$-Laplacian on time scales. Bound. Value Probl. 2018, Article ID 70 (2018)

12. Dong, X., Bai, Z., Zhang, S.: Positive solutions to boundary value problems of $p$-Laplacian with fractional derivative. Bound. Value Probl. 2017, Article ID 5 (2017)

13. Tian, Y., Sun, S., Bai, Z:: Positive solutions of fractional differential equations with $p$-Laplacian. J. Funct. Spaces 2017 , Article ID 3187492 (2017)

14. Yuan, C.: Multiple positive solutions for $(n-1,1)$-type semipositone conjugate boundary value problems of nonlinear fractional differential equations. Electron. J. Qual. Theory Differ. Equ. 36, 1 (2010)

15. He, L., Dong, X., Bai, Z., Chen, B.: Solvability of some two-point fractional boundary value problems under barrier strip conditions. J. Funct. Spaces 2017, Article ID 1465623 (2017)

16. Zuo, M., Hao, X., Liu, L., Cui, Y.: Existence results for impulsive fractional integro-differential equation of mixed type with constant coefficient and antiperiodic boundary conditions. Bound. Value Probl. 2017, Article ID 161 (2017)

17. Bai, Z., Dong, X., Yin, C.: Existence results for impulsive nonlinear fractional differential equation with mixed boundary conditions. Bound. Value Probl. 2016, Article ID 63 (2016)

18. Sun, Q., Ji, H., Cui, Y.: Positive solutions for boundary value problems of fractional differential equation with integral boundary conditions. J. Funct. Spaces 2018, Article ID 6461930 (2018)

19. Song, Q., Bai, Z.: Positive solutions of fractional differential equations involving the Riemann-Stieltjes integral boundary condition. Adv. Differ. Equ. 2018, Article ID 183 (2018) 
20. Zou, Y., He, G.: The existence of solutions to integral boundary value problems of fractional differential equations at resonance. J. Funct. Spaces 2017, Article ID 2785937 (2017)

21. Ma, W., Meng, S., Cui, Y.: Resonant integral boundary value problems for Caputo fractional differential equations. Math. Probl. Eng. 2018, Article ID 5438592 (2018)

22. Ma, W., Cui, Y.: The eigenvalue problem for Caputo type fractional differential equation with Riemann-Stieltjes integral boundary conditions. J. Funct. Spaces 2018, Article ID 2176809 (2018)

23. Ahmad, B., Luca, R.: Existence of solutions for a sequential fractional integro-differential system with coupled integral boundary conditions. Chaos Solitons Fractals 104, 378-388 (2017)

24. Qi, T., Liu, Y., Zou, Y.: Existence result for a class of coupled fractional differential systems with integral boundary value conditions. J. Nonlinear Sci. Appl. 10, 4034-4045 (2017)

25. Qiu, X., Xu, J., O'Regan, D., Cui, Y.: Positive solutions for a system of nonlinear semipositone boundary value problems with Riemann-Liouville fractional derivatives. J. Funct. Spaces 2018, Article ID 7351653 (2018)

26. Hao, X., Wang, H., Liu, L., Cui, Y.: Positive solutions for a system of nonlinear fractional nonlocal boundary value problems with parameters and p-Laplacian operator. Bound. Value Probl. 2017, Article ID 182 (2017)

27. Qi, T., Liu, Y., Cui, Y.: Existence of solutions for a class of coupled fractional differential systems with nonlocal boundary conditions. J. Funct. Spaces 2017, Article ID 6703860 (2017)

28. Zhang, Y.: Existence results for a coupled system of nonlinear fractional multi-point boundary value problems at resonance. J. Inequal. Appl. 2018, Article ID 198 (2018)

29. Zhang, X., Liu, L., Zou, Y.: Fixed-point theorems for systems of operator equations and their applications to the fractional differential equations. J. Funct. Spaces 2018, Article ID 7469868 (2018)

30. Zhang, X., Liu, L., Wu, Y., Zou, Y.: Existence and uniqueness of solutions for systems of fractional differential equations with Riemann-Stieltjes integral boundary condition. Adv. Differ. Equ. 2018, Article ID 204 (2018)

31. Li, H., Zhang, J.: Positive solutions for a system of fractional differential equations with two parameters. J. Funct. Spaces 2018, Article ID 1462505 (2018)

32. Zhao, Y., Hou, X., Sun, Y., Bai, Z:: Solvability for some class of multi-order nonlinear fractional systems. Adv. Differ. Equ. 2019, Article ID 23 (2019)

33. Asawasamrit, S., Ntouyas, S., Tariboon, J.: Coupled systems of sequential Caputo and Hadamard fractional differential equations with coupled separated boundary conditions. Symmetry 10(2), Article ID 701 (2018)

34. Yang, W.: Positive solutions for singular coupled integral boundary value problems of nonlinear Hadamard fractional differential equations. J. Nonlinear Sci. Appl. 8(2), 110-129 (2015)

35. Zhai, C., Wang, W. Li, H.: A uniqueness method to a new Hadamard fractional differential system with four-point boundary conditions. J. Inequal. Appl. 2018, Article ID 207 (2018)

36. Zhang, K., Fu, Z:: Solutions for a class of Hadamard fractional boundary value problems with sign-changing nonlinearity. J. Funct. Spaces 2019, Article ID 9046472 (2019)

37. Zhang, K., Wang, J., Ma, W.: Solutions for integral boundary value problems of nonlinear Hadamard fractional differential equations. J. Funct. Spaces 2018, Article ID 2193234 (2018)

38. Benhamida, W., Graef, J.R., Hamani, S.: Boundary value problems for Hadamard fractional differential equations with nonlocal multi-point boundary conditions. Fract. Differ. Calc. 8(1), 165-176 (2018)

39. Benchohra, M., Bouriah, S., Nieto, J.J.: Existence of periodic solutions for nonlinear implicit Hadamard's fractional differential equations. Rev. R. Acad. Cienc. Exactas Fís. Nat., Ser. A Mat. 112(1), 25-35 (2018)

40. Wang, G., Pei, K., Agarwal, R.P., Zhang, L.: Nonlocal Hadamard fractional boundary value problem with Hadamard integral and discrete boundary conditions on a half-line. J. Comput. Appl. Math. 343, 230-239 (2018)

41. Tariboon, J., Cuntavepanit, A., Ntouyas, S.K., Nithiarayaphaks, W.: Separated boundary value problems of sequential Caputo and Hadamard fractional differential equations. J. Funct. Spaces 2018, Article ID 6974046 (2018)

42. Huang, H., Liu, W.: Positive solutions for a class of nonlinear Hadamard fractional differential equations with a parameter. Adv. Differ. Equ. 2018, Article ID 96 (2018)

43. Matar, M.M.: Solution of sequential Hadamard fractional differential equations by variation of parameter technique. Abstr. Appl. Anal. 2018, Article ID 9605353 (2018)

44. Yang, W.: Monotone iterative technique for a coupled system of nonlinear Hadamard fractional differential equations. J. Appl. Math. Comput. in press

45. Abbas, S., Benchohra, M., Hamidi, N., Henderson, J.: Caputo-Hadamard fractional differential equations in Banach spaces. Fract. Calc. Appl. Anal. 21(4), 1027-1045 (2018)

46. Vivek, D., Kanagarajan, K., Elsayed, E.M.: Nonlocal initial value problems for implicit differential equations with Hilfer-Hadamard fractional derivative. Nonlinear Anal., Model. Control 23(3), 341-360 (2018)

47. Ahmad, B., Ntouyas, S.K.: Nonlocal initial value problems for Hadamard-type fractional differential equations and inclusions. Rocky Mt. J. Math. 48(4), 1043-1068 (2018)

48. Belhannache, F., Hamani, S., Henderson, J.: Upper and lower solutions methods for impulsive Caputo-Hadamard fractional differential inclusions. Electron. J. Differ. Equ. 2019, 22, 1-13 (2019)

49. Kilbas, A.A., Srivastava, H.M., Trujillo, J.J.: Theory and Applications of Fractional Differential Equations. Elsevier, Boston (2006)

50. Guo, D., Lakshmikantham, V.: Nonlinear Problems in Abstract Cones. Academic Press, Orlando (1988) 